\title{
Summary of session C1: experimental gravitation
}

\author{
C Lämmerzahl \\ ZARM, University of Bremen, Am Fallturm, 28359 Bremen, Germany \\ E-mail: laemmerzahl@zarm.uni-bremen.de
}

Received 29 October 2007, in final form 21 November 2007

Published 15 May 2008

Online at stacks.iop.org/CQG/25/114023

\begin{abstract}
The fact that gravity is a metric theory follows from the Einstein equivalence principle. This principle consists of (i) the universality of free fall, (ii) the universality of the gravitational redshift and (iii) the local validity of Lorentz invariance. Many experiments searching for deviations from standard general relativity test the various aspects of the Einstein equivalence principle. Here we report on experiments covering the whole Einstein equivalence principle. Until now all experiments have been in agreement with the Einstein equivalence principle. As a consequence, gravity has to be described by a metric theory. Any metric theory of gravity leads to effects such as perihelion shift, deflection of light, gravitational redshift, gravitational time delay, Lense-Thirring effect, Schiff effect, etc. A particular theory of that sort is Einstein's general relativity. For weak gravitational fields which are asymptotically flat any deviation from Einstein's general relativity can be parametrized by a few constants, the PPN parameters. Many astrophysical observations and space experiments are devoted to a better measurement of the effects and, thus, of the PPN parameters. It is clear that gravity is best tested for intermediate ranges, that is, for distances between $1 \mathrm{~m}$ and several astronomical units. It is highly interesting to push forward our domain of experience and to strengthen the experimental foundation of gravity also beyond these scales. This point is underlined by the fact that many quantum gravity and unification-inspired theories suggest deviation from the standard laws of gravity at very small or very large scales. In this session summary we briefly outline the status and report on the talks presented in session $\mathrm{C} 1$ about experimental gravitation.
\end{abstract}

PACS numbers: $04.20 .-\mathrm{q}, 04.80 . \mathrm{Cc}, 04.90 .+\mathrm{e}, 95.40 .+\mathrm{s}$

\section{The importance and basis of relativistic gravity}

The meaning and importance of relativity and gravity can be summarized in the statement that it is the physics of space and time. Since all physical phenomena have to happen within 
space and time relativistic gravity is at the very basis of all physics. Space and time have to be measured and explored by clocks and moving objects. Correspondingly, at the end all tests of relativistic gravity are based on clocks and on the observation of the motion of particles.

This is also mirrored by the basic principle underlying general relativity, the Einstein equivalence principle (EEP). This principle consists of three parts: the principle of the universality of free fall (UFF), also called the weak equivalence principle, the principle of the universality of the gravitational redshift (UGR), also called local position invariance, and the local validity of Lorentz invariance (LLI). The validity of the EEP implies that gravity has to be described by a pseudo-Riemannian metric. If one of these principles fails to hold, then there will be more gravitational field than just a spacetime metric.

UFF states that two different pointlike neutral particles move along the same spacetime trajectory when released at the same spacetime point with the same initial velocity. This is a pure orbit comparison test. UGR states that the rates of two different clocks (which are not allowed to be based on the interaction with an external gravitational field like a pendulum) do not depend on the position of these clocks. Finally, LLI is based on the comparison of clocks having different orientations and velocities. Therefore, these merely orbit and clock comparison tests imply that gravity is metric. One particular metric theory is Einstein's general relativity (GR). If one of these tests fails then GR will no longer hold.

Any metric theory of gravity predicts effects which are not present in the Newtonian theory and which are related to the rates of clocks and trajectories. These effects are light bending, perihelion shift, gravitational redshift, a gravitational time delay and the LenseThirring effect. The Schiff effect, the precession of gyroscopes in a gravitomagnetic field is an effect of the motion of an extended particle which can be derived from the motion of point particles with restricted degrees of freedom. A further predicted effect is gravitational waves whose detection is also related to the motion of test particles, the mirrors and light rays. Einstein's theory of relativity is then characterized by a certain magnitude of these effects.

Accordingly, there are two possibilities for deviations from GR: (i) within the class of metrical theories but with different magnitudes of effects. Examples of that are all theories which are related to non-standard PPN parameters, see e.g. a list given in [1]. (ii) non-metric theories of gravity. In this case there are additional fields, in general tensor fields of any rank, related to gravitational phenomena. Examples of these are theories with torsion, with a non-metricity or with a Finslerian metric.

In the following we first describe these tests in more detail and point to the corresponding talks held in the session $\mathrm{C} 1$.

\section{Tests of the universality of free fall}

UFF applies to neutral point-like particles only. The corresponding tests are described in terms of the acceleration of these particles in the reference frame of the gravitating body: the Eötvös factor compares the normalized accelerations of two bodies $\eta=\frac{a_{1}-a_{2}}{\frac{1}{2}\left(a_{1}+a_{2}\right)}$ in the same gravitational field. In the frame of Newtons theory this can be expressed as $\eta=\frac{\mu_{1}-\mu_{2}}{\frac{1}{2}\left(\mu_{1}+\mu_{2}\right)}$ with $\mu=m_{\mathrm{g}} / m_{\mathrm{i}}$ where $m_{\mathrm{g}}$ is the gravitational and $m_{\mathrm{i}}$ the inertial mass, respectively. Though there are no point particles it is possible experimentally to manufacture macroscopic bodies such that their higher gravitational multipoles either are very small or very well under control. This is used in the various tests of the UFF.

There are hints from quantum gravity inspired scenarios that the UFF might be violated below the $10^{-13}$ level [2, 3]. Also from cosmology with a dynamical vacuum energy (quintessence) one can derive a violation of the UFF at the $10^{-14}$ level [4]. The validity 
of the UFF has also been used for posing bounds on the time variability of various constants such as the fine structure constant and the electron-to-proton mass ratio [5].

In session $\mathrm{C} 1$ there was one presentation dedicated to a forthcoming UFF test. H Selig (Bremen) on behalf of H Dittus, M List, S Grotjan and S Theil reported on the principle and new developments of the mission MICROSCOPE for testing the UFF in space [6]. The accuracy aimed for is $10^{-15}$, thus being more than two orders of magnitude better than corresponding tests on Earth. This mission is approved and scheduled for 2011. The focus of this talk was on the dynamics and error estimate of the accelerometers, one of the central parts of this mission.

We know from lunar laser ranging that the gravitational self-energy of the Earth-Moon system falls toward the Sun in the same way as ordinary matter at the same rate to within $10^{-3}$. The question of whether the gravitational constant $G$ may depend on the temperature can be interpreted as whether kinetic energy falls in a gravitational field in the same way as ordinary matter. This has been discussed by A Dmitriev (St Petersburg) [7].

E Rosenthal (Cornell) together with E E Flanagan examined the generalization of the Hayashi-Shirafuji theory suggested by Mao et al [8] called Einstein-Hayashi-Shirafuji theory. They showed that for any coupling to matter that is compatible with the spin transport equation postulated in [8] the theory has either ghosts, tachyons or an ill-posed initial value formulation. These theoretical problems can be avoided by specializing the parameters of the theory and in addition choosing the standard minimal coupling to matter of the torsion tensor. This yields a consistent theory, but one in which the action equals reaction principle is violated, and in which the angular momentum of gyroscopes does not couple to the Earth's torsion field. Thus, the Einstein-Hayashi-Shirafuji theory does not predict a detectable torsion signal for Gravity Probe B [9].

\section{Tests of the universality of the gravitational redshift}

For a test of this principle the run of clocks based on different physical principles has to be compared during their common transport through a gravitational potential. There are a large variety of clocks: (i) light clocks, (ii) atomic clocks based on hyperfine transitions, (iii) on fine structure transitions, (iv) on principal transitions, (v) molecular clocks based on rotational transitions, (vi) on vibrational transitions, (vii) gravitational clocks based on revolution of planets or binary systems, (viii) pulsar clocks based on the spin of stars, and (ix) clocks based on particle decay.

On a phenomenological level the comparison of the two collocated clocks is given by

$$
\frac{v_{1}\left(x_{1}\right)}{v_{2}\left(x_{1}\right)} \approx\left(1-\left(\alpha_{\text {clock} 2}-\alpha_{\text {clock } 1}\right) \frac{U\left(x_{1}\right)-U\left(x_{0}\right)}{c^{2}}\right) \frac{v_{1}\left(x_{0}\right)}{v_{2}\left(x_{0}\right)},
$$

where $\alpha_{\text {clocki }}$ are clock-dependent parameters. If this frequency ratio does not depend on the gravitational potential then the gravitational redshift is universal. This is a null-test of the quantity $\alpha_{\text {clock } 2}-\alpha_{\text {clock } 1}$. It is obviously preferable to use a large difference in the gravitational potential which shows the need for space experiments. In experiments today the variation of the gravitational field is induced by the motion of the Earth around the Sun that requires the used clocks to have very good long-term stability.

There is no test so far for 'anti clocks', that is, clocks made of anti-matter. However, since the production of anti-hydrogen is a well working technique today, there are attempts to perform high-precision spectroscopy of anti-hydrogen. These measurements should first test special relativistic CPT invariance but, as a long-term measurement, can also be used to test the UGR for a clock based on anti-hydrogen. 
The best test to date has been performed by comparing the frequency ratio of the $282 \mathrm{~nm}$ ${ }^{199} \mathrm{Hg}^{+}$optical clock transition to the ground state hyperfine splitting in ${ }^{133} \mathrm{Cs}$ over 6 years. The result is $[10,11]\left|\alpha_{\mathrm{Hg}}-\alpha_{\mathrm{Cs}}\right| \leqslant 5 \times 10^{-6}$ and was presented by $\mathrm{N}$ Ashby (Colorado) for T P Heavner, S R Jefferts, T E Parker, A G Radnaev and Y O Dudin. Other tests compare Cs clocks with the hydrogen maser, $\mathrm{Cs}$ or electronic transitions in $\mathrm{I}_{2}$ with optical resonators.

A Sfarti (Berkeley) proposed the use of a mine in South Africa in order to do controlled experiments within a mine shaft which is $4 \mathrm{~km}$ deep. For that height even second-order effects should be observable with high precision clocks. However, we are looking forward to ultrastable clocks on the ISS and on satellites.

\section{Tests of local Lorentz invariance}

The first step to Lorentz invariance is the confirmation that the velocity of light $c$ is unique. This means first that $c$ does not depend on the velocity of the source. With the model $c^{\prime}=c+\kappa v$, where $v$ is the velocity of the source (in some frame) and $\kappa$ some parameter, astrophysical observations give $\kappa \leqslant 10^{-11}$ [12] while laboratory experiments yield $\kappa \leqslant 10^{-6}$ [13]. Second, all experiments and observations report that all other massless particles and the limiting velocity of all massive particles coincide with $c$. Therefore, the velocity of light is a universal structure and can be interpreted as part of the geometrical structure of spacetime.

All the following tests can be regarded as tests with clocks, searching for a dependence of the clock experiments on the orientation or the velocity. In the optical sector such dependences could be restricted to be smaller than $10^{-16}$ reported in $[14,15]$. There are two high precision experiments with different cavity materials, different cavity geometries and different operating frequencies at different locations, namely Berlin and Perth. M Tobar (Perth) and his collaborators P L Stanwix, E N Ivanov, A C Fowler, J G Hartnett, J-M G le Floch, M M Niao and P Wolf are members of the above collaboration which his work significantly contributed to. He reported about their latest results from two complementary tests of Lorentz invariance in electrodynamics. The first test is an even parity test, which compares two orthogonal cryogenic sapphire microwave oscillators rotating in the lab. They acquired over one year of data and are able to place independent limits on all eight SME parameters improving previous measurements by a factor of 10 . Within the RobertsonMansouri-Sexl framework the limit on the isotropy parameter of $\leqslant 9.4 \times 10^{-11}$ improved by a factor of 2 . The second test is a rotating odd parity test, which compares the phase shifts of the two one-way propagating waves that experience different permeabilities over the length of propagation. A sensitive carrier suppression microwave interferometer is used to obtain highly sensitive phase comparison using a Mach-Zehnder configuration. This experiment is sensitive to the isotropic Lorentz violating parameter and is the first rotating experiment of this type.

W-T Ni (Nanjing) described possible tests of Lorentz invariance on astrophysical and cosmological scales. A pseudoscalar-photon interaction would induce a rotation of linear polarization of electromagnetic wave propagating with cosmological/astrophysical distance. In 2003, WMAP observed the correlation of polarization with temperature anisotropy in the cosmic microwave background. From the alignment of this correlation with the principal axes of the quadrupole temperature anisotropy in WMAP observation, one can put a limit of $0.1 \mathrm{rad}$ on the rotation of linear polarization of cosmological microwave background propagation. Pseudoscalar-photon interaction is proportional to the gradient of the 
pseudoscalar field. From a phenomenological point of view, this gradient could be a neutrino number asymmetry [18], another density current or a constant vector. Then Lorentz invariance or CPT may effectively be violated $[16,17]$.

Finally, H-H Mei (Hsinchu) described a number of experiments that are underway to detect vacuum birefringence and dichroism, namely: (i) PVLAS (polarization of vacuum with LASers), (ii) quantum-electrodynamics and axion (Q\&A), and (iii) BMV (Biréfringence Magnétique du Vide). Recently, PVLAS observed an optical rotation in vacuum by a magnetic field (vacuum dichroism). Theoretical interpretations of this result include a possible pseudoscalar-photon interaction and the existence of millicharged fermions. Mei described his Q\&A experiment for vacuum dichroism and vacuum birefringence which is developing together with W-T Ni, S-J Chen and S-S Pan [19]. With the obtained sensitivity it would be possible to check the polarization rotation effect obtained by PVLAS.

\section{Test of general relativity}

Besides the tests of the foundations of metrical theories of gravity we describe here experiments which test whether the metrical theory is GR or deviates from it. Within a post-Newtonian approximation such deviations may show up in the Newtonian potential. A deviation of the Newtonian potential from the standard $1 / r$ form at short or at large distances clearly would signal a new metrical theory of gravity beyond GR. In fact, deviations from the ordinary $1 / r$ potential are the consequence of suggested explanations of various problems in the standard theory. These problems are, e.g., (i) the hierarchy problem in particle physics addressing the fact that the electromagnetic interaction is of about 40 orders of magnitude larger than the gravitational interaction, (ii) the problem of the cosmological constant which is by about 120 orders of magnitude smaller than predicted from particle physics, and (iii) the strong PC puzzle. Solutions to (i) may come from higher dimensional models [20], to (ii) from a cutting off the gravitational interaction at $\sim 50 \mu \mathrm{m}$ [21], and (iii) may be solved by postulating a new particle, the axion, which is also a candidate for dark matter. In any of these models a deviation from the $1 / r$-potential is suggested.

Long scale deviations from the $1 / r$ law have been suggested in higher dimensional braneworld scenarios [22] and also in scenarios with a running coupling constant [23].

C Speake (Birmingham) in cooperation with G D Hammond, C Trenkel and A PulidoPaton set new constraints on short-range forces which couple mass to intrinsic spin. They used a spherical superconducting torsion balance and placed new upper limits on forces that couple mass to intrinsic spin. Their experimental approach uses a novel spin source geometry that allows unprecedented sensitivity in the range $100 \mu \mathrm{m}<\lambda<5 \mathrm{~mm}$. They placed new limits on the dimensionless coupling constant of such an interaction of $g_{p}^{e} g_{s} \leqslant\left(-1.9 \pm 1.3_{\text {stat }} \pm 1.5_{\text {syst }}\right) \cdot 10^{-26}$ for $\lambda>10 \mathrm{~mm}$ at $1 \sigma$ confidence level. At a range of $1 \mathrm{~mm}$ their most relaxed limit is $g_{p}^{e} g_{s} \leqslant 1.5 \times 10^{-24}$ [24].

H-J Paik (Maryland) working together with V Prieto, M V Moody and K Venkateswara is conducting a sub-millimeter test of the $1 / r^{2}$ law at $1.7 \mathrm{~K}$. To minimize Newtonian errors, the experiment employs a near-null source, a circular disc of large diameter-to-thickness ratio. Two test masses, also disc-shaped, are positioned on the two sides of the source mass at a nominal distance of $150 \mu \mathrm{m}$. The test masses are connected to a SQUID to form a superconducting differential accelerometer. As the source is driven sinusoidally, a violation signal should appear at the second harmonic due to symmetry. In order to avoid a tilt and recoil of the platform and distortion of the housing, a rotating source mass would alleviate these problems and allow the masses to be aligned more precisely, leading to a more sensitive 
experiment at $\leqslant 100 \mu \mathrm{m}$. The detector could consist of two disc-shaped levitated test masses. A short-range force from the rotating source would exert a stronger periodic torque on the near test mass than on the far test mass. This signal could be detected with a superconducting differential angular accelerometer. See also [25].

In order to test the large scale behavior of gravity one has to go to space. In fact, there are many proposals which aim at a test of large scale gravity: ASTROD, LATOR, ODYSSEY, SAGAS, among others. Furthermore, there was already a large scale gravity mission with the spacecraft Pioneer 10 and 11. This is indeed the largest man-made experiment ever carried through. There were two presentations by H Dittus (Bremen) describing the ASTROD mission (with W-T Ni et al) and activities concerning the analysis of the old Pioneer data and the concept for a new Pioneer mission.

The mission ASTROD (Astrodynamic Space Test of Relativity using Optical Devices) is a large scale mission of two spacecraft at distances of approx $1 \mathrm{AU}$ which will be ranged from Earth [26]. The mission objectives are: (i) testing relativistic gravity with five orders of magnitude improvement, (ii) improving the sensitivity in the $5 \mu \mathrm{Hz}$ to $5 \mathrm{mHz}$ low frequency range for gravitational-wave detection, (iii) increasing the sensitivity of solar, planetary and asteroid parameter determination by three to four orders of magnitude, (iv) detection of solar $g$-mode oscillations. This will require a post-post-Newtonian ephemeris framework to be established for the analysis and simulation of data.

Dittus (with cooperators C Lämmerzahl, M List, L Mullin and S Theil) also reported on the analysis of the radiometric tracking data of the Pioneer 10 and 11 spacecraft which indicated an anomalous Doppler frequency drift between heliocentric distances between 20 and 70 astonomical units [27]. The uniformingly changing blueshift can be interpreted as a constant Sunward acceleration. The nature of the so-called Pioneer Anomaly is still not clear. Explanation attempts are mainly dedicated to understanding the temporal and spatial constancy. All conventional mechanisms for an anomalous acceleration show strong temporal or spatial variations. Dittus outlined the new analysis of the complete Pioneer data started at ZARM, University of Bremen, and also the progress toward a thermal model of the Pioneer spacecraft.

R Woodard (Gainsville) together with E O Kahya considered alternate gravity models, such as Bekenstein's TeVeS, that succeed in emulating galactic rotation curves and weak lensing without dark matter. Powerful theoretical constraints on such models predispose ordinary matter to travel along different geodesics than gravitons. Hence there will be a time lag between the pulse of gravitons from a supernova and the subsequent neutrino and optical pulses. They showed that this time lag depends only upon the supernova position, not on the details of the alternate gravity model [28]. For a repeat of SN 1987a the time lag would be several days.

Finally, M Tajmar reported an effect near rotating superconductors which has the signature of a gravitomagnetic field [29] which he observed with F Plesescu and B Seifert. This effect is 18 orders of magnitude larger than Einstein's theory predicts. It has been discussed whether the origin of the effect is some vibration inside the whole setup. These measurements have been repeated at the ring laser gyroscope in Christchurch, New Zealand. No such effects have been seen [30]. There is also the claim that the observed effect might explain the misalignment torques in the GP-B experiment. However, if it did, then the observed misalignment torques on the GP-B gyroscopes, which are proportional to the misalignment between the satellite roll axis and the gyroscope spin axis, would have been constant throughout the measurement period. In fact, the observed misalignment torques appear to be correlated with the changing polhode path of the spin axis in each of the gyroscopes. Therefore, even if the observed effect is a true effect, then it could not explain the GP-B measurements [31]. 


\section{New results/interpretation within GR}

One of the most important issues today is the direct detection of gravitational waves. A lot of experimental effort for their detection on ground as well as in spacer has been spent. First, it is important to verify directly this prediction of GR. Even more important is the information about the physics, the structure and the dynamics of compact binary objects. Since the two-particle system cannot be solved analytically in GR, one can either use post-Newtonian approximation methods, or one can study extreme cases like the one if one member of the binary system is very large. Then the small companion can be treated nearly as a test particle. The emitted radiation then leads the smaller particle to approach the larger companion, thus leading to extreme mass ratio inspirals (EMRI). The question now is how one can extract from the detected gravitational waves information about the gravitational field. This can also be extended to intermediate mass ratio inspirals (IMRI).

The form of the observed gravitational wave contains the information about the geodesics of the smaller companion, and in this geodesics the information about the gravitational field is encoded. The question is how exactly to extract that information. This was the content of the contribution by J Brink (Caltech) about orbits in stationary spacetimes. One important question addressed in her lecture was whether geodesic equations are integrable. A lot of new developments and progress is expected if one is able to find besides the ordinary constants of motion and Carter's constant a forth invariant for the geodesic motion.

It has also been speculated whether gravity may have some influence on quantum scales. K Rosquist (Stockholm) emphasized that for Einstein-Maxwell equations the tendency of the gravitational field to additionally contribute to electromagnetic effects increases as the size of the system goes down. This has consequences for the electromagnetic fields of spinning charged particles. He obtained corrections to the Coulomb force for charged spinning sources. Experimentally verifiable consequences include an electric quadrupole moment for the electron, possible quasi-bound states in positron-heavy ion scattering with sizes corresponding to observed anomalous peaks, as well as small corrections to energy levels in microscopic bound systems such as the hydrogen atom [32]. Until now, radiative effects have not yet been taken into account.

\section{New technologies}

In this section, we describe new technologies for better tests of gravitational physics and also for better technical applications. These technical applications are gyroscopes and accelerometers which may have ground applications like the mapping of gravity but also space applications like formation flying, gravitational experiments in space, autonomous operations of satellite constellations, and planetary exploration. Conventional gyroscopes today work with laser light and accelerometers with freely falling test masses. The rotation of the laboratory influences the phase shift of a laser interferometer and the acceleration of test masses also can be read off by interferometric methods. By the upcoming of atomic interferometry both apparatuses can be combined in one atomic interferometer-both the rotation and the acceleration induce phase shifts. Furthermore, due to the mass of the atoms, the sensitivities of these devices are much higher than the conventional devices.

The recent development in this area has been reported by L Mondin (Toulouse) for P Bouyer, A Landragin et al. She talked about recent progress in the development of an atomic interferometer for application as inertial sensor in space: ICE (Interférométrie Cohérente pour l'Espace), see [33, 34]. In space this inertial sensor can serve as an accelerometer for navigation of satellites but also as a device for measuring the frame dragging effect or, when operated 
with different atomic species, for performing tests of the UFF. For the latter the operation in space is crucial: while on Earth the interrogation time is limited by the time given by the available fall height, performance in space allows for longer free fall and, thus, for enhanced precision. A free fall test of this device has been performed during a zero- $g$ parabolic flight of an Airbus.

S Shiomi (Hsinchu) presented applications of superconducting gravimeters to test gravitational physics [35]. Superconducting gravimeters are proved to be sensitive and stable in geophysical studies. They are the most sensitive instruments to measure surface gravity changes at low frequencies. Currently, more than 20 superconducting gravimeters are operating in the world and a global network has been developed. She discussed the possible effects of non-Newtonian gravity which could be searched for with these devices and also showed that the UFF can be tested on Earth down to a level of $10^{-9}$. Improvements can be expected from combinations of global measurements and advanced data analysis.

$\mathrm{N}$ Ashby presented the method of side-ranging Laser Imaging Detection and Ranging (LIDAR) to measure wind from space. This method will probably use a laser transmitter in a spacecraft to illuminate aerosol particles or molecules that scatter the radiation almost exactly back toward the transmitter. The measured frequency shift can be processed to yield the velocity of the scattering particles relative to the ground. Ashby discussed relativistic corrections to the determination of the wind velocity, arising from large spacecraft velocities, Earth rotation and acceleration of the spacecraft [36].

K-X Sun (Stanford) in cooperation with S Buchman, G Allan, R L Byer, J Conklin, D Debra, S Higuchi, N Leindecker, P Lu and A Swank presented technologies for an advanced modular gravitational reference sensor (MGRS). This MGRS is based on the concept of the sensor as a 'black box' independent of the rest of the experiment. He presented the recent progress, including a gravitational wave detector configuration with a single spherical proof mass per corner for the flight formation, and in-field telescope pointing.

\section{Acknowledgments}

I would like to thank all the speakers in session $\mathrm{C} 1$ for their helpful cooperation. The financial support of the German Aerospace Center DLR is acknowledged.

\section{References}

[1] Will C M 1993 Theory and Experiment in Gravitational Physics revised edn (Cambridge: Cambridge University Press)

[2] Damour T, Piazza F and Veneziano G 2002 Phys. Rev. Lett. 89081601

[3] Damour T, Piazza F and Veneziano G 2002 Phys. Rev. D 66046007

[4] Wetterich C 2003 Astropart. Phys. 102

[5] Dent T 2006 Varying 'constants' in astrophysics and cosmology Preprint hep-ph/0610376

[6] Touboul P, Rodrigues M, Metris G and Tatry B 2001 C. R. Acad. Sci. Ser IV: Phys., Astrophys. 21271

[7] Dmitriev A L, Nikushchenko E M and Snegov V S 2003 Meas. Tech. 46115

[8] Mao Y, Tegmark M, Guth A and Cabi S 2007 Phys. Rev. D 76104029

[9] Flanagan E E and Rosenthal E 2007 Phys. Rev. D 75124016

[10] Ashby N et al 2007 Phys. Rev. Lett. 98070802

[11] Fortier T M et al 2007 Phys. Rev. Lett. 98070801

[12] Brecher K 1977 Phys. Rev. Lett. 391051

[13] Amelino-Camelia G et al 2005 The search for quantum gravity signals Gravitation and Cosmology Proc. 758th AIP Conf. (Melville, New York) ed A Macias, C Lämmerzahl and D Nunez p 30

[14] Stanwix P L et al 2005 Phys. Rev. D 74081101

[15] Müller H et al 2007 Phys. Rev. Lett. 99050401 
[16] Ni W-T 2005 Chin. Phys. Lett. 2233

[17] Feng B et al 2006 Phys. Rev. Lett. 96221302

[18] Geng C Q et al 2007 Preprint 0706.0080

[19] Chen S-J, Mei H-H and Ni W-T 2006 Q \& A experiment to search for vacuum dichroism, pseudoscalar-photon interaction and millicharged fermions Preprint hep-ex/0611050v1

[20] Arkani-Hamed N, Dimopoulos S and Dvali G 1998 Phys. Lett. B 429263

[21] Sundrum R 1999 J. High Energy Phys. JHEP07(1999)001 Sundrum R 2004 Phys. Rev. D 69044014

[22] Dvali D, Gabadadze G and Porrati M 2000 Phys. Lett. B 485208

[23] Jaekel M-T and Reynaud S 2005 Mod. Phys. Lett. A 201047 Jaekel M-T and Reynaud S 2005 Class. Quant. Grav. 222135

[24] Hammond G D et al 2007 Phys. Rev. Lett. 98081101

[25] Paik H J, Moody M V and Strayer D M 2004 Gen. Rel. Grav. 36523

[26] Huang T-Y et al (guest eds) 2002 Int. J. Mod. Phys. D 11 947-1158

[27] Anderson J D et al 2002 Phys. Rev. D 65082004

[28] Kahya E O and Woodard R P 2007 Phys. Lett. B 652 213-6

[29] Tajmar M et al 2007 Preprint 0707.3806v5

[30] Graham G et al Experiment to detect frame-dragging in a lead superconductor http://www2.phys.canterbury. ac.nz/ physrin/papers/SuperFrameDragging2007.pdf

[31] Keiser G 2007 private communication (mac@ relgyro.stanford.edu)

[32] Rosquist K 2006 Class. Quantum Grav. 233111

[33] Bouyet P et al 2007 Atom interferometric sensors for applications in space Lasers, Clocks and Drag-Free: Exploration of Relativistic Gravity in Space ed H Dittus, C Lämmerzahl and S G Turyshev (Berlin: Springer) p 297

[34] Varoquaux G et al 2007 ICE: new transportable atom inertial sensor for applications to space experiments Proc. Moriond Meeting at press

[35] Shiomi S 2006 Phys. Rev. D 74027101

[36] Ashby N 2007 J. Opt. Soc. Am. 243530 